\title{
Scaling Limit of Some Critical Models
}

\author{
J.-R. Fontaine \\ Institut de Physique Théorique, Université Catholique de Louvain, Chemin du Cyclotron, 2, \\ B-1348 Louvain-la-Neuve. Belgium*
}

\begin{abstract}
In this paper we consider massless systems which are strong perturbations of the massless lattice free field. Under quite general assumptions on the potential, we prove that the continuum (scaling) limit of these systems is Gaussian.
\end{abstract}

\section{Introduction}

In this paper we study the (continuum) scaling limit of some massless models of classical statistical mechanics. The main particularity of the systems considered is that their correlations are not absolutely summable; typically they have a clustering like $|x|^{-d}$ in $d$ dimensions. Such a situation arises for the low temperature rotator model [1] or for the lattice dipole gas [2]. These systems are rather well approximated by the following model of an anharmonic crystal, defined on $\mathbb{Z}^{d}$ and described by the Hamiltonian [3]:

$$
H=\frac{1}{2} \sum_{x}\left(\nabla_{x} \phi\right)^{2}+\lambda / 4 \sum_{x}\left(\nabla_{x} \phi\right)^{4},
$$

where $\phi_{x}$ is a real random variable uniformly distributed on the real line. For small coupling $\lambda$ the (block spin) scaling limit of this model can be obtained using the machinery of Gawedzki and Kupiainen based on rigorous renormalization group arguments as announced in [4] (see also [5] for related results by Magnen and Sénéor). For large coupling $\lambda$, the question of the scaling limit is, so far, totally open.

In [6] we developed a method essentially based on correlation inequalities to obtain bounds (uniform in $\lambda$ ) on the long distance behaviour of general correlation functions of model (1). We feel that those bounds should be useful to study the scaling limit of this model for arbitrary $\lambda$.

In this paper we consider a simplification of model (1): the Hamiltonian that we choose is

$$
H=\frac{1}{2} \sum_{x}\left(\nabla_{x} \phi\right)^{2}+\lambda / 4 \sum_{x}\left((-\Delta)^{\alpha} \phi_{x}\right)^{4},
$$

\footnotetext{
* New address: Institut de Physique Theorique, Ecole Polytechnique Fédérale de Lausanne,
} PHB, CH-1015 Lausanne, Switzerland 
where $\Delta$ is the lattice Laplacian and $\alpha>\frac{1}{2}$. Using the method of [6], we are able to show that for arbitrary $\lambda$, the (continuum) scaling limit of this model is Gaussian. In the case $\alpha>\frac{1}{2}$, the dielectric constant of the system is $\lambda$-independent. This is precisely the fact which makes this problem simpler than the case $\alpha=\frac{1}{2}$ [which is equivalent to model (1)]. Indeed, in the case of model (1) the dielectric constant is renormalized by the interaction, see $[6,5]$. We finally want to mention that model (2) has been studied in the weak coupling limit by Malyshev and Tirozzi [7], and Federbush $[8,9]$.

The paper is organised as follows: in Sect. 2 we give a precise definition of the model and of its properties, in Sect. 3 we present the results, and in Sect. 4 we give the proofs.

\section{The Model and its Properties}

\subsection{Definition of the Model}

Let $\phi_{x}\left(x \in \mathbb{Z}^{d}, d \geqq 3\right)$ be a real random variable. To each region $\Lambda$ ( $\Lambda$ is a parallelipiped of side lengths $2 l_{1}, \ldots, 2 l_{d}$ ) is associated an Hamiltonian $H_{\Lambda}$ with periodic boundary conditions on $\partial \Lambda$ :

$$
H_{\Lambda}=\frac{1}{2} \sum_{\substack{x \in \Lambda \\ \xi}}\left(\nabla_{x}^{\xi} \phi\right)+\lambda / 4 \sum_{x \in \Lambda}\left(\left(-\Delta_{\Lambda}\right)^{\alpha} \phi_{x}\right)^{4}
$$

where

$$
\nabla_{x}^{\xi} \phi=\phi\left(x+e_{\xi}\right)-\phi(x), \quad\left\{e_{\xi}\right\}_{\xi=1}^{d}
$$

is the canonical basis of $\mathbb{Z}^{d}\left(e_{\xi}=\delta_{i \xi}, i=1, \ldots, d\right)$. Here $\left(-\Delta_{\Lambda}\right)^{\alpha} \equiv \delta_{\Lambda}$ is defined by $\delta_{\Lambda} \phi_{x}$ $=\sum_{y} \beta_{\Lambda}(x-y) \phi_{y}$

$$
\beta_{\Lambda}(x)=(2 \pi)^{-d}|\Lambda|^{-1} \sum_{p \in \Lambda^{*}}[\operatorname{expip} x]\left[2 \sum_{\xi}\left(1-\cos p_{\xi}\right)\right]^{\alpha} .
$$

$p_{\xi} \equiv p \cdot e_{\xi}, \Lambda^{*}$ is the dual lattice associated to $\Lambda$, i.e.

$$
\left\{p \mid p_{\xi}=2 \pi n_{\xi} / l_{\xi} ; n_{\xi}=-l_{\xi},-l_{\xi}+1, \ldots, l_{\xi}\right\},
$$

and $|\Lambda|$ is the cardinality of $\Lambda$.

We also introduce the Hamiltonian:

$$
H_{\Lambda, m}=H_{\Lambda}+m^{2} / 2 \sum_{x \in \Lambda} \phi_{x}^{2} \quad(m \geqq 0) .
$$

For $m \neq 0$ expectation values of functions $g$ of the type $\prod_{i \in A} \phi_{i}$ are defined via

where

$$
\langle g\rangle_{\Lambda, m}=Z_{\Lambda, m}^{-1} \int_{\mathbb{R}} g \exp -H_{\Lambda, m} \prod_{x \in \Lambda} d \phi_{x},
$$

$$
Z_{\Lambda}=\int_{\mathbb{R}^{|\Lambda|}} \exp -H_{\Lambda, m} \prod_{i \in \Lambda} d \phi_{i}
$$

Here $A$ is a "subset" of $A$; in order to avoid exponents we allow repetitions of the same element in $A$, which is why we used the word subset in quotation marks. 


\subsection{Brascamp-Lieb (B-L) Inequalities and Definition of the States}

2.2.1. Definition. A function $F: \mathbb{R}^{n} \rightarrow \mathbb{R}_{+}$is $\log$ concave if $F(\phi)=\exp f(\phi)$, and $f$ is concave. (It is understood that $f$ can take the value $-\infty$.)

2.2.2. Let $A$ be a strictly positive symmetric $n \times n$ matrix, and denote by \langle\rangle$_{0}$ the Gaussian measure associated to it:

$$
\langle\rangle_{0}=\left[\int \exp \left[-\frac{1}{2}(\Phi, A \Phi) d \Phi\right]^{-1} \int \cdot \exp \left[-\frac{1}{2}(\Phi, A \Phi)\right] d \Phi ;\right.
$$

consider a perturbation of \langle\rangle$_{0}$ by a log concave function $F$ :

$$
\langle\rangle_{F}=\left[\int \exp \left[-\frac{1}{2}(\Phi, A \Phi)\right] F(\Phi) d \Phi\right]^{-1} \int \cdot \exp \left[-\frac{1}{2}(\Phi, A \Phi)\right] F(\Phi) d \Phi ;
$$

$\phi_{x}, x \in\{1, \ldots, n\}$ denotes a component of $\Phi$. We can now state the basic theorem:

2.2.3. Proposition [10]. The covariance matrix $M_{x y}^{F}=\left\langle\phi_{x} \phi_{y}\right\rangle_{F}-\left\langle\phi_{x}\right\rangle_{F}\left\langle\phi_{y}\right\rangle_{F}$ satisfies the inequality $M_{x y}^{F} \leqq\left(A^{-1}\right)_{x y}$ in the sense of forms. (This will be denoted by B-L inequalities.)

$(-\lambda) \sum_{x \in \Lambda}\left(\delta_{\Lambda} \phi_{x}\right)^{4}$ is a concave function on $\mathbb{R}^{|\Lambda|}$, because the matrix of its second derivatives is positive. For any $h: \Lambda \rightarrow \mathbb{R}$,

$$
\begin{array}{rl}
\sum_{x, y} & h(x) h(y) \frac{\partial}{\partial \phi_{x}} \frac{\partial}{\partial \phi_{y}}\left[\sum_{z \in \Lambda}\left(\delta_{\Lambda} \phi_{x}\right)^{4}\right] \\
& =12 \sum_{x, y} h(x) h(y)\left[\sum_{z \in \Lambda}\left(\delta_{\Lambda} \phi_{z}\right)^{2} \beta_{\Lambda}(x-z) \beta_{\Lambda}(y-z)\right] \\
& =12 \sum_{z \in \Lambda}\left[\left(\delta_{\Lambda} \phi_{z}\right)^{2}\left(\sum_{x} \beta_{\Lambda}(x-z) h(x)\right)^{2}\right] \geqq 0 .
\end{array}
$$

We can therefore apply Brascamp-Lieb inequalities to show:

$$
\left\langle[\phi(f)]^{2}\right\rangle_{\Lambda, m} \leqq \sum_{x, y} f(x) f(y) C_{\Lambda}^{m}(x, y),
$$

where $\phi(f)=\sum_{x} f(x) \phi_{x}$, and $f: \Lambda \rightarrow \mathbb{R} ; C_{\Lambda}^{m}(x, y)$ is the kernel of the operators $\left(-\Delta_{\Lambda}\right.$ $\left.+m^{2}\right)^{-1}$.

We shall use the notations $C^{m}(x, y)=\lim _{\Lambda \rightarrow \infty} C_{\Lambda}^{m}(x, y)$ and $C^{m=0}(x, y)=C(x, y)$. Because of the properties of the Gaussian model and because of (2.2.3), one can define the limits (possibly via subsequences)

$$
\lim _{m \downarrow 0} \lim _{\Lambda \uparrow \infty}\langle g\rangle_{\Lambda, m} \equiv\langle g\rangle(d \geqq 3) .
$$

For $d<3$ the limiting state is only defined on functions of the type $\prod_{(x, \xi) \in A}\left(\nabla_{x}^{\xi} \phi\right)$, where $A$ is a "subset" of $A \times\left\{e_{1}, \ldots, e_{d}\right\}$. For more details see [3]. In what follows $\left\langle>\right.$ will denote any of the limiting states obtained from (3). If $f$ is a real $l^{2}$ function defined on $\mathbb{Z}^{d}$, its Fourier transform is defined by:

$$
f^{\wedge}(p)=\sum_{x} f(x) \exp i p x
$$




\subsection{Bounds on the Two Point Function}

We mention two bounds on the Fourier transform of the two point function $S(p) \equiv\left\langle\phi_{0} \phi_{x}\right\rangle^{\wedge}(p)$. The upper bound is a rewriting of Proposition 2.2.2; the lower bound is a kind of Mermin-Wagner bound [11].

\subsubsection{Proposition}

(ii)

$$
\begin{gathered}
S(p) \leqq\left[2 \sum_{e=1}^{d}\left(1-\cos p_{e}\right)\right]^{-1}, \\
S(p) \geqq\left[2 \sum_{e=1}^{d}\left(1-\cos p_{e}\right)\right]^{-1}-3 \lambda w(p),
\end{gathered}
$$

where

$$
w(p)=\left[2 \sum_{e=1}^{d}\left(1-\cos p_{e}\right)\right]^{2 \alpha-2}\left\langle\left(\delta \phi_{0}\right)^{2}\right\rangle,
$$

which is finite in any dimension.

2.3.2. Remark. (5) is based on integration by parts formula that we now recall.

Let $\left\{\phi_{z}\right\}_{z=1}^{n}$ be a set of Gaussian random variables and $\mu$ be the corresponding Gaussian measure with covariance $C_{x y}^{\prime}$. We have the formula (see [12])

$$
\int \phi_{y} F\left(\left\{\phi_{z}\right\}\right) d \mu=\sum_{x=1}^{n} C_{x y}^{\prime} \int \frac{d}{d \phi_{x}} F\left(\left\{\phi_{z}\right\}\right) d \mu .
$$

2.3.3. We introduce the set

$$
\mathscr{A}=\left\{f: \mathbb{Z}^{d} \rightarrow \mathbb{R} \mid \sum_{x, y} f(x) f(y) C(x, y)<\infty\right\} .
$$

2.3.4. Proof of 2.3.1 (ii). As we shall see, it is convenient to introduce the notation $c * \beta=\gamma$. For any $f$ in $\mathscr{A}$, after using (6) twice, we have:

$$
\begin{aligned}
\sum_{x, y} f(x) f(y)\left\langle\phi_{x} \phi_{y}\right\rangle & \\
= & \sum_{x, y} f(x) f(y) C(x, y) \\
& \quad-3 \lambda \sum_{z, x, y} f(x) f(y) \gamma(x-z) \gamma(y-z)\left\langle\left(\delta \phi_{z}\right)^{2}\right\rangle \\
& +\lambda^{2} \sum_{z, z^{\prime}, x, y} f(x) f(y) \gamma(x-z) \gamma\left(y-z^{\prime}\right)\left\langle\left(\delta \phi_{z}\right)^{3}\left(\delta \phi_{z^{\prime}}\right)^{3}\right\rangle .
\end{aligned}
$$

The third term of the right hand side of (7) can be written as

$$
\lambda^{2}\left\langle\left[\sum_{x, z} f(x) \gamma(x-z)\left(\delta \phi_{z}\right)^{3}\right]^{2}\right\rangle \geqq 0
$$

\section{The Results}

\subsection{Definition of the Continuum Scaling Limit}

3.1.1. For any integer $\theta>1$, we introduce the scaled fields:

$$
\phi_{x}^{\theta} \equiv \alpha(\theta) \phi_{\theta x}
$$


where $\alpha(\theta)$ is a function of the scale parameter $\theta$, that will be specified later, and

$$
x \in \mathbb{Z}_{\theta^{-1}}^{d}=\left\{y \in \mathbb{R}^{d} \mid \theta y \in \mathbb{Z}^{d}\right\} .
$$

The scaled correlation functions are given by:

$$
G_{\theta}\left(x_{1}, \ldots, x_{n}\right) \equiv \alpha(\theta)^{n}\left\langle\phi_{\theta x_{1}} \ldots \phi_{\theta x_{n}}\right\rangle ;
$$

$\alpha(\theta)$ is chosen such that $\lim _{\theta \rightarrow \infty} G_{\theta}\left(x_{1}, \ldots, x_{n}\right)$ exists. The weak limit of the random fields $\phi_{x}^{\theta}$ as $\theta \rightarrow \infty$ is called the continuum scaling limit of the theory. We refer to [13-16] for an introduction to the concept of scaling limit and for other definitions.

Given any $f \in \mathscr{S}\left(\mathbb{R}^{d}\right)$ (the Schwartz space on $\mathbb{R}^{d}$ ), we shall denote its restriction to $\mathbb{Z}_{\theta^{-1}}^{d}$, by $f^{\theta}$. It is convenient to smear out the scaled fields :

$$
\phi^{\theta}\left(f^{\theta}\right) \equiv \sum_{x \in \mathbb{Z}_{\theta-1}^{d}} \theta^{-d} \phi_{x}^{\theta} f^{\theta}(x) .
$$

Equation (8) can be rewritten as:

$$
\begin{aligned}
\phi^{\theta}\left(f^{\theta}\right) & =\sum_{x \in \mathbb{Z}_{\theta^{-1}}^{d}} \theta^{-d} \alpha(\theta) \phi_{x} f^{\theta}\left(\theta^{-1} x\right) \\
& =\phi\left(f_{\theta}\right),
\end{aligned}
$$

where $f_{\theta}(x)=\theta^{-d} \alpha(\theta) f^{\theta}\left(\theta^{-1} x\right)$.

For obvious reasons we shall always use the notation $\phi\left(f_{\theta}\right)$ instead of $\phi^{\theta}\left(f^{\theta}\right)$.

3.1.2. Scaling Limit of the Lattice Gaussian Free Field. In the case $\lambda=0$, it is easy to show that the scaling limit exists; we have to choose $\alpha(\theta)=\theta^{(d-2) / 2}$. Define

$$
\begin{aligned}
& f^{\theta \wedge}(k)=\theta^{-d} \sum_{x \in \mathbb{Z}_{\theta^{-1}}^{d}} f(x) \exp (i k x), \quad k \in[-\pi \theta \pi \theta], \\
& \sum_{x, y \in \mathbb{Z}^{d}} \theta^{-2 d} \theta^{d-2} C(x, y) f^{\theta}\left(\theta^{-1} x\right) f^{\theta}\left(\theta^{-1} y\right) \\
& =\theta^{d-2}(2 \pi)^{-d} \int_{-\pi}^{\pi}\left|f^{\theta \wedge}(p)\right|^{2}\left[2 \sum_{e}\left(1-\cos p_{e}\right)\right]^{-1} d^{d} p .
\end{aligned}
$$

After scaling $p \theta \rightarrow p$, this becomes

$$
(2 \pi)^{-d} \int_{-\pi \theta}^{\pi \theta}\left|f^{\theta \wedge}(p)\right|^{2}\left[\theta^{2} 2 \sum_{e}\left(1-\cos p_{e}\right)\right]^{-1} d^{d} p,
$$

which converges to

$$
\begin{gathered}
2(2 \pi)^{-d} \int_{-\infty}^{+\infty}\left|f^{\wedge}(p)\right|^{2} p^{-2} d^{d} p \equiv(f, D f) \\
f^{\wedge}(p)=\int_{-\infty}^{+\infty} f(x) \exp (\text { ipx } x) d^{d} p .
\end{gathered}
$$

This shows that the continuum scaling limit of the lattice massless free field is the continuum massless free field. 
3.1.3. Remark. A similar computation would show:

$$
\lim _{\theta \rightarrow \infty} \sum_{x, y \in \mathbb{Z}^{d}} C^{\varepsilon}(x, y) f_{\theta}(x) f_{\theta}(y)=0,
$$

where $C^{\varepsilon}(x, y)$ denotes the kernel of the operator

$$
(-\Delta)^{-1+\varepsilon} \quad(\varepsilon>0) \text {. }
$$

3.1.4. The main result of the paper is that the continuum scaling limit of the theory with $\lambda$ arbitrary is the continuum Gaussian free field. Again in this case $\alpha(\theta)$ $=\theta^{(d-2) / 2}$. This is expressed in the

3.2. Theorem. Choose $\alpha(\theta)=\theta^{(d-2) / 2}$. For any $\lambda>0$, and any $f \in \mathscr{S}\left(\mathbb{R}^{d}\right)$ :

(i) $\lim _{\theta \rightarrow \infty}\left\langle\left[\phi\left(f_{\theta}\right)\right]^{2}\right\rangle=(f, D f)$,

(ii) $\lim _{\theta \rightarrow \infty}\left\langle\left[\phi\left(f_{\theta}\right)\right]^{2 n}\right\rangle=2 n !\left(n ! 2^{n}\right)^{-1}(f, D f)^{n}$.

\subsection{Remarks}

3.3.1. Even though all our results have been formulated for $d \geqq 3$, they extend trivially for any $d$. In the case $d \leqq 2$, we have to use the scaled fields $\nabla \phi_{x}^{\theta}=\theta^{d / 2}\left(\phi_{\theta x+1}\right.$ $\left.-\phi_{\theta x}\right)$.

3.3.2. Our results also extend to more general interactions: $\sum_{x} \lambda\left(\delta \phi_{x}\right)^{4}$ can be for instance replaced by $\lambda P\left(\left(\delta \phi_{x}\right)^{2}\right)$, where $P$ is a polynomial with positive coefficients.

\section{The Proofs}

As will be shown below, Theorem 3.2(i) is a direct consequence of Proposition 2.3.1. To derive part (ii) we shall use methods developed in [6], where we were able to combine Brascamp-Lieb inequalities and the method of duplicated variables to get bounds of the type

$$
\sum_{x, y} f(x) f(y)\left[\left\langle\left(\nabla_{x} \phi\right)^{n}\left(\nabla_{y} \phi\right)^{n}\right\rangle-\left\langle\left(\nabla_{0} \phi\right)^{n}\right\rangle^{2}\right] \leqq c\|f\|_{l^{2}},
$$

where $c$ is a $\lambda$-independent positive constant.

Caution. In the whole paper, $c$ will denote a positive constant which can take different values at different places.

\subsection{Proof of Theorem 3.2(i)}

Using Proposition 2.3.1 we obtain the bounds:

(i) $\left\langle\phi\left(f_{\theta}\right)^{2}\right\rangle \leqq \sum_{x, y \in \mathbb{Z}^{d}} C(x, y) f_{\theta}(x) f_{\theta}(y)$,

(ii) $\left\langle\phi\left(f_{\theta}\right)^{2}\right\rangle \geqq \sum_{x, y \in \mathbb{Z}^{d}} f_{\theta}(x) f_{\theta}(y)\left[C(x, y)-3 \lambda\left\langle\left(\delta \phi_{0}\right)^{2}\right\rangle C^{\varepsilon}(x, y)\right]$,

with $\varepsilon=2 \alpha-1>0$. Section 3.1.2 and Remark 3.1.3 imply that, in the limit $\theta \rightarrow \infty$, the upper and lower bounds coincide with $(f, D f)$. 


\subsection{The Scaling Limit of the $2 n$ Points Functions}

4.2.1. The main part of the paper concerns the proof of Theorem 3.2(ii). It is based on two propositions, the first one being obtained using methods developed in [6] that we now recall. Let us introduce a new set of variables $\left\{\phi_{x}^{\prime}, x \in \mathbb{Z}^{d}\right\}$, which is just a duplication of the set $\left\{\phi_{x}, x \in \mathbb{Z}^{d}\right\}$. Consider the unnormalized density

$$
\exp \left\{\sum_{z}\left[-\frac{1}{2}\left(\phi_{z},-\Delta \phi_{z}\right)-\lambda / 4\left(\delta \phi_{z}\right)^{4}-\frac{1}{2}\left(\phi_{z}^{\prime},-\Delta \phi_{z}^{\prime}\right)-\lambda / 4\left(\delta \phi_{z}^{\prime}\right)^{4}\right]\right\} \text {. }
$$

Using the variables

$$
\begin{aligned}
& \psi_{z}^{+}=\frac{1}{2}\left(\phi_{z}+\phi_{z}^{\prime}\right), \\
& \psi_{z}^{-}=\frac{1}{2}\left(\phi_{z}-\phi_{z}^{\prime}\right),
\end{aligned}
$$

(9) becomes

$$
\begin{aligned}
G\left(\psi^{+}, \psi^{-}\right)= & \exp \left\{2 \sum _ { z } \left[-\frac{1}{2}\left(\psi_{z}^{+},-\Delta \psi_{z}^{+}\right)\right.\right. \\
& -\lambda / 4\left(\delta \psi_{z}^{+}\right)^{4}-\frac{3}{2} \lambda\left(\delta \psi_{z}^{+}\right)^{2}\left(\delta \psi_{z}^{-}\right)^{2} \\
& \left.\left.-\frac{1}{2}\left(\psi_{z}^{-},-\Delta \psi_{z}^{-}\right)-\lambda / 4\left(\delta \psi_{z}^{-}\right)^{4}\right]\right\}
\end{aligned}
$$

$\langle>$ will denote the normalized measure associated with (10). We also introduce the notation:

$$
H\left(\psi^{+}, \psi^{-}\right)=\exp \left\{2 \sum_{z}\left[-\frac{1}{2}\left(\psi_{z}^{-},-\Delta \psi_{z}^{-}\right)-\lambda / 4\left(\delta \psi_{z}^{-}\right)^{4}-\frac{3}{2} \lambda\left(\delta \psi_{z}^{+}\right)^{2}\left(\delta \psi_{z}^{-}\right)^{2}\right]\right\} .
$$

For any $f$ and $g \in \mathscr{A}$,

$$
\begin{aligned}
& \psi^{ \pm}(f) \equiv \sum_{x \in \mathbb{Z}^{d}} \psi_{z}^{ \pm} f(z), \\
& \psi^{ \pm}(f)^{2 n} \equiv\left[\psi^{ \pm}(f)\right]^{2 n} .
\end{aligned}
$$

4.2.2. Proposition. For any $f, g \in \mathscr{A}$ :

$$
\begin{aligned}
& \left\langle\psi^{+}(f)^{2 n} \psi^{-}(g)^{2}\right\rangle-\left\langle\psi^{+}(f)^{2 n}\right\rangle\left\langle\psi^{-}(g)^{2}\right\rangle \\
& \leqq 3 \lambda\left\langle\psi^{+}(f)^{2 n}\right\rangle \int\left|g^{\wedge}(p)\right|^{2} w(p) d^{d} p
\end{aligned}
$$

where $w(p)$ is defined in (2.3.1)

Proof. $\left\langle\psi^{+}(f)^{2 n} \psi^{-}(g)^{2}\right\rangle$ can be written as :

$$
\begin{aligned}
& \int d \psi^{+} \psi^{+}(f)^{2 n}\left\{d \psi^{-} \psi^{-}(g)^{2} H\left(\psi^{+}, \psi^{-}\right)\left(\int d \psi^{-} H\left(\psi^{+}, \psi^{-}\right)\right)^{-1}\right\} \\
& \cdot \int d \psi^{-} G\left(\psi^{+}, \psi^{-}\right) Z_{\Lambda}^{-1} .
\end{aligned}
$$

We used the notations: $d \psi^{ \pm}=\prod_{x \in \Lambda} d \psi_{x}^{ \pm}$, and $Z_{\Lambda}=\int d \psi^{+} d \psi^{-} G\left(\psi^{+}, \psi^{-}\right)$. For any configuration of $\psi^{+}$,

$$
2 \sum_{z}\left[\lambda / 4\left(\delta \psi_{z}^{-}\right)^{4}+\frac{3}{2} \lambda\left(\delta \psi_{z}^{-}\right)^{2}\left(\delta \psi_{z}^{+}\right)^{2}\right]
$$

is convex. Therefore, using Brascamp-Lieb inequality (2.2.3), we have: 


$$
\left\langle\psi^{+}(f)^{2 n} \psi^{-}(g)^{2}\right\rangle \leqq \sum_{x, y} \frac{1}{2} C(x, y) g(x) g(y)\left\langle\psi^{+}(f)^{2 n}\right\rangle
$$

So

$$
\begin{aligned}
& \left\langle\psi^{+}(f)^{2 n} \psi^{-}(g)^{2}\right\rangle-\left\langle\psi^{+}(f)^{2 n}\right\rangle\left\langle\left(\psi^{-}(g)^{2}\right\rangle\right. \\
& \quad \leqq \sum_{x, y} \frac{1}{2} g(x) g(y)[C(x, y)-\langle\phi(x) \phi(y)\rangle]\left\langle\psi^{+}(f)^{2 n}\right\rangle .
\end{aligned}
$$

Now using the lower bound on the two point function of Proposition 2.3.1, this last inequality finishes the proof 4.2.2(i).

4.2.3. Remark. In the case of a purely Gaussian theory $(\lambda=0)$ we have

$$
\left\langle\psi^{+}(f)^{2 n} \psi^{-}(g)^{2}\right\rangle=\left\langle\psi^{+}(f)^{2 n}\right\rangle\left\langle\psi^{-}(g)^{2}\right\rangle,
$$

and this translated into the $\phi$ and $\phi^{\prime}$ variables, expresses $\left\langle\phi(f)^{2 n+2}\right\rangle$ in terms of $\left\langle\phi(f)^{2 k}\right\rangle(2 \leqq k \leqq 2 n)$. Therefore using this relation in a recursive way we get Wick's theorem.

4.2.4. We shall first use Proposition 4.2.2 to show $\left\langle\left[\phi\left(f_{\theta}\right)\right]^{2 n}\right\rangle \leqq c(f, n)$, where $c(f, n)$ is $f$ and $n$ dependent but $\theta$ independent. This last result will be used in Proposition 4.2.7 to get a lower bound on $\left\langle\left[\phi\left(f_{\theta}\right)\right]^{2 n}\right\rangle$. Finally combining Proposition 4.2.2 and 4.2.7 with an induction, we shall prove Theorem 3.2(ii).

\subsubsection{Corollary.}

$$
\forall f \in \mathscr{S}\left(\mathbb{R}^{d}\right) \text { and } \forall n \in \mathbb{N}, \quad\left\langle\phi\left(f_{\theta}\right)^{2 n}\right\rangle \leqq c(f, n),
$$

where $c(f, n)$ is $\theta$ independent.

Proof. The proof will follow from an induction on $n$. Let $P(m)$ be the proposition: $\left\langle\phi\left(f_{\theta}\right)^{2 p}\right\rangle \leqq c(f, p)$ for $1 \leqq p \leqq m$. Assume $P(m)$, we want to prove $P(m+1)$. Proposition 4.2.2 expressed in the $\phi$ variables yields:

$$
\begin{aligned}
\left\langle\phi\left(f_{\theta}\right)^{2 m+2}\right\rangle \leqq & \sum_{\substack{b, c=1, \ldots, m \\
d=0,1}} \alpha_{b c d}\left\langle\phi\left(f_{\theta}\right)^{2 b}\right\rangle\left\langle\phi\left(f_{\theta}\right)^{2 c}\right\rangle\left\langle\phi\left(f_{\theta}\right)^{2 d}\right\rangle \\
& +\left[\sum_{p=0}^{m} 4^{-m}\left(\begin{array}{c}
2 m \\
2 p
\end{array}\right)\left\langle\phi\left(f_{\theta}\right)^{2 m-2 p}\right\rangle\left\langle\phi\left(f_{\theta}\right)^{2 p}\right\rangle\right] \\
& \cdot \frac{3}{2} \lambda \int_{-\pi}^{\pi}\left|f^{\theta \wedge}(p)\right|^{2} w(p) d^{d} p,
\end{aligned}
$$

where $\alpha_{b c d}$ are positive numerical factors [we have dropped the negative terms that should appear in the right hand side of (12)].

Using the induction hypothesis we get:

$$
\left\langle\phi\left(f_{\theta}\right)^{2 m+2}\right\rangle \leqq c_{1}(f, 2 m+2)+c_{2} \frac{3}{2} \lambda \theta^{d-2} \int_{-\pi}^{\pi}\left|f^{\theta \wedge}(p)\right|^{2} w(p) d^{d} p .
$$

Remark 3.1.3 implies that the second term of the right hand side of (13) goes to zero as $\theta \rightarrow \infty$. Therefore (13) yields $P(m+1)$.

Since the induction hypothesis is true for $m=1$ [it is Theorem 3.2, Part (i)], the corollary is proven. 
4.2.6. Remark. As a direct consequence of Proposition 4.2.2, Corollary 4.2.5, and Remark 3.1 .3 we have:

$$
\lim _{\theta \rightarrow \infty}\left[\left\langle\psi^{+}\left(f_{\theta}\right)^{2 n} \psi^{-}\left(f_{\theta}\right)^{2}\right\rangle-\left\langle\psi^{+}\left(f_{\theta}\right)^{2 n}\right\rangle\left\langle\psi^{-}\left(f_{\theta}\right)^{2}\right\rangle\right] \leqq 0 .
$$

4.2.7. Proposition. $\lim _{\theta \rightarrow \infty}\left\langle\phi\left(f_{\theta}\right)^{2 n}\right\rangle \geqq 2 n !\left(n ! 2^{n}\right)^{-1}(f, D f)^{n}$.

Proof. Using the integration by parts formula (2.3.2),

$$
\begin{aligned}
&\left\langle\phi\left(f_{\theta}\right)^{2 n}\right\rangle=(2 n-1)\left(f_{\theta}, C f_{\theta}\right)\left\langle\phi\left(f_{\theta}\right)^{2 n-2}\right\rangle \\
&-\lambda \sum_{z, x} f_{\theta}(x) \gamma(x-z)\left\langle\phi\left(f_{\theta}\right)^{2 n-1}\left(\delta \phi_{z}\right)^{3}\right\rangle, \\
&\left(\left(f_{\theta}, C f_{\theta}\right) \equiv \sum_{x, y} f_{\theta}(x) f_{\theta}(y) C(x-y)\right) .
\end{aligned}
$$

It is convenient to use the notations

$$
\begin{aligned}
g(z) \equiv \sum_{x} f_{\theta}(x) \gamma(x-z) ; \quad g^{\wedge}(p) & =\theta^{(d-2) / 2} f^{\theta \wedge}(p \theta)\left[2 \sum_{e}\left(1-\cos p_{e}\right)\right]^{\alpha-1} \\
(\delta \phi)^{3}(g) & \equiv \sum_{z} g(z)\left(\delta \phi_{z}\right)^{3}
\end{aligned}
$$

By Schwartz inequality:

$$
\left\langle\phi\left(f_{\theta}\right)^{2 n}\right\rangle \geqq(2 n-1)\left(f_{\theta}, C f_{\theta}\right)\left\langle\phi\left(f_{\theta}\right)^{2 n-2}\right\rangle-\lambda\left\langle\phi\left(f_{\theta}\right)^{4 n-2}\right\rangle^{1 / 2}\left\langle\left[(\delta \phi)^{3}(g)\right]^{2}\right\rangle^{1 / 2} .
$$

We now remark three facts:

(i) $\left(f_{\theta}, C f_{\theta}\right) \geqq\left\langle\phi\left(f_{\theta}\right)^{2}\right\rangle$

by Brascamp-Lieb inequality.

(ii) $\left\langle\phi\left(f_{\theta}\right)^{4 n-2}\right\rangle^{1 / 2} \leqq c$,

where $c$ is a $\theta$-independent constant (see Corollary 4.2.5).

(iii) $\lim _{\theta \rightarrow \infty}\left\langle\left[(\delta \phi)^{3}(g)\right]^{2}\right\rangle^{1 / 2}=0$.

This is a consequence of the following argument:

$$
\sum_{x, y} g(x) g(y)\left\langle(\delta \phi)^{3}(x)(\delta \phi)^{3}(y)\right\rangle \leqq c\|g\|_{l_{2}}^{2},
$$

where $c$ is $\lambda$-independent; this result can be derived as in [6].

Finally, $\lim _{\theta \rightarrow \infty}\|g\|_{l_{2}}^{2}=0$ [see (14) and Remark 3.1.3].

Inequality (15) and (i)-(iii) imply:

$$
\lim _{\theta \rightarrow \infty}\left\langle\phi\left(f_{\theta}\right)^{2 n}\right\rangle \geqq(2 n-1)\left\langle\phi\left(f_{\theta}\right)^{2}\right\rangle\left\langle\phi\left(f_{\theta}\right)^{2 n-2}\right\rangle .
$$

A recursive application of (16) yields Proposition 4.2.7.

4.2.8. Proof of Theorem 3.2.(ii). The proof is by induction on $n$. Let us first remark that Theorem 3.2(ii) is true for $n=1$ since this statement is Theorem 3.2(i). Let $P(p)$ 
be the following property:

$$
\lim _{\theta \rightarrow \infty}\left\langle\phi\left(f_{\theta}\right)^{2 n}\right\rangle=2 n !\left(n ! 2^{n}\right)^{-1}(f, D f),
$$

for $1 \leqq n \leqq p$. Given $P(p)$ we want to show that $P(p+1)$ is true. Using Remark 4.2.6, we have:

$$
\lim _{\theta \rightarrow \infty}\left[\left\langle\psi^{+}\left(f_{\theta}\right)^{2 p} \psi^{-}\left(f_{\theta}\right)^{2}\right\rangle-\left\langle\psi^{+}\left(f_{\theta}\right)^{2 p}\right\rangle\left\langle\psi^{-}\left(f_{\theta}\right)^{2}\right\rangle\right] \leqq 0 .
$$

Going back to the $\phi$ variables, this yields :

$$
\begin{aligned}
\lim _{\theta \rightarrow \infty}\left\langle\phi\left(f_{\theta}\right)^{2 p+2}\right\rangle \leqq & \lim _{\theta \rightarrow \infty} \sum_{\begin{array}{r}
c . d=1, \ldots, p \\
c+d=p \\
e=0,1
\end{array}} \beta_{c d e} \\
& \cdot\left\{\left\langle\phi\left(f_{\theta}\right)^{2 c}\right\rangle\left\langle\phi\left(f_{\theta}\right)^{2 d}\right\rangle\left\langle\phi\left(f_{\theta}\right)^{2 e}\right\rangle\right\},
\end{aligned}
$$

where $\beta_{c d e}$ are numerical factors, positive or negative. If we now use the induction hypothesis to

$$
\lim _{\theta \rightarrow \infty}\left\langle\phi\left(f_{\theta}\right)^{2 c}\right\rangle\left\langle\phi\left(f_{\theta}\right)^{2 d}\right\rangle
$$

we have:

$$
\lim _{\theta \rightarrow \infty}\left\langle\phi\left(f_{\theta}\right)^{2 p+2}\right\rangle \leqq c\left\langle\phi\left(f_{\theta}\right)^{2}\right\rangle^{p+1} .
$$

Note that $c$ is independent of $\lambda$, and that for $\lambda=0$ (17) has to be an equality; therefore $c=(2 p+2) ! /\left[(p+1) ! 2^{p+1}\right]$. We thus have:

$$
\lim _{\theta \rightarrow \infty}\left\langle\phi\left(f_{\theta}\right)^{2 p+2}\right\rangle \leqq\left[(2 p+2) ! /\left((p+1) ! 2^{p+1}\right)\right] \lim _{\theta \rightarrow \infty}\left\langle\phi\left(f_{\theta}\right)^{2}\right\rangle^{p+1}
$$

this combined with Proposition 4.2.7 proves the validity of the induction hypothesis $P(p+1)$.

4.2.9. Remarks. Consider a field theory (in dimension $d$ ) described by a set of Schwinger functions satisfying the Osterwalder-Schrader axioms [17]. Suppose that the field theory is a scaling limit of some lattice theory. Newman showed that if the two point (Schwinger) function $S(x)$ of the field theory behaves like $|x|^{-(d-2)}$ for large $x$, then it is a massless free field [18]. In our case we do not know a priori whether the scaling limit of the model we consider exists and satisfies the Osterwalder-Schrader axioms. So even though we know that the scaling limit of the two point function has the required behaviour, we cannot use the result described above. However, our proof has the same spirit as Newman's theorem. In particular Proposition 4.2.2 shows that the correction to Wick's formula is bounded by the difference between the scaling limit of $S(p)$ and the free massless two point function.

Acknowledgements. I would like to thank the referee and J. Fröhlich for suggesting to consider the continuum scaling limit instead of the block spin scaling limit, as was done in a previous version of the paper. 


\section{References}

1. Bricmont, J., Fontaine, J.-R., Lebowitz, J., Lieb, E., Spencer, T.: Lattice systems with a continuous symmetry. III. Low temperature asymptotic expansion for the plane rotator model. Commun. Math. Phys. 78, 545 (1981)

2. Fontaine, J.-R.: Low fugacity asymptotic expansion for classical lattice dipole gases. J. Stat. Phys. 26, 767 (1981)

3. Bricmont, J., Fontaine, J.-R., Lebowitz, J., Spencer, T.: Lattice systems with a continuous symmetry. I. Perturbation theory for unbounded spins. Commun. Math. Phys. 78, 281 (1980)

4. Gawedzki, K., Kupiainen, A. : Block renormalization group for dipole gas and $(\nabla \phi)^{4}$. University of Helsinki (preprint)

5. Magnen, J., Sénéor, R.: The infrared behaviour of $(\nabla \phi)_{3}^{4}$. Preprint Centre de Physique Théorique de l'Ecole Polytechnique, Palaiseau

6. Fontaine, J.-R.: Bounds on the decay of correlations for $(\nabla \phi)^{4}$ models. Commun. Math. Phys. 87, 385 (1982)

7. Malyshev, V.A., Tirozzi, B.: Renormalization group convergence for small perturbations of Gaussian random fields with slowly decaying correlations. J. Math. Phys. 22, 2020 (1981)

8. Federbush, P.: A mass zero cluster expansion. Part. 1. The expansion. Commun. Math. Phys. 81, $327(1981)$

9. Federbush, P.: A mass zero cluster expansion. Part. 2. Convergence. Commun. Math. Phys. 81, 341 (1981)

10. Brascamp, H.J., Lieb, E.H.: On extensions of the Brunn-Minkowski and Prekopa-Leindler theorems, including inequalities for Log concave functions, and with an application to the diffusion equation. J. Funct. Anal. 22, 366 (1976)

11. Mermin, N.D., Wagner, H.: Absence of ferromagnetism or antiferromagnetism in one- or twodimensional isotropic Heisenberg models. Phys. Rev. Lett. 17, 1133 (1966)

12. Glimm, J., Jaffe, A., Spencer, T.: The particle structure of the weakly coupled $P(\phi)_{2}$ model and other applications of high temperature expansions. In: Constructive quantum field theory. Velo, G., Wightman, A. (eds.). Lecture Notes in Physics, Vol. 25. Berlin, Heidelberg, New York : Springer 1973

13. Glimm, J., Jaffe, A.: Particles and scaling for lattice fields and Ising models. Commun. Math. Phys. 51, 1 (1976)

14. Newman, C.M.: Normal fluctuations and the FKG inequalities. Commun. Math. Phys. 74, 119 (1980)

15. Fröhlich, J., Spencer, T.: Some recent rigorous results in the theory of phase transitions and critical phenomena. Séminaire Bourbaki No. 586 (February 1982)

16. Sinai, Ya.G.: Mathematical foundations of the renormalization group method in statistical physics. In: Mathematical problems in theoretical physics. Dell'Antonio, G., Doplicher, S., JonaLasinio, G. (eds.). Lecture Notes in Physics, Vol. 80. Berlin, Heidelberg, New York: Springer 1978

17. Osterwalder, K., Schrader, R.: Axioms for Euclidean Green's functions. Commun. Math. Phys. 31, 83-112 (1973)

18. Newman, C.M.: Self-similar random fields in mathematical physics. Proceedings of the measure theory conferences. Northern Illinois University, Dekalb, IL. (1980)

Communicated by J. Fröhlich

Received April 11, 1983; in revised form June 21, 1983 
